\title{
Procesos de magnetización reversible-irreversible y propiedades de remanencia de ferrita de cobalto coprecipitada
}

\author{
A. MEDINA-BOUDRI', D. BUENO-BAQUÉS' ${ }^{2}$ J. MATUTES-AQUINO' \\ ${ }^{1}$ Centro de Investigación en Materiales Avanzados. Complejo Industrial Chihuahua Miguel de Cervantes 120, Chihuahua, Chih., Mexico \\ ${ }^{2}$ Laboratorio de Magnetismo, Facultad de Física-IMRE, Universidad de la Habana, La Habana 10400, Cuba.
}

Se obtuvo ferrita de cobalto por coprecipitación de sulfatos de hierro y cobalto en acetona. Usando el modelo móvil de Preisach se separaron las componentes reversible $\left(M_{r e v}\right)$ e irreversible $\left(M_{i r r}\right)$ de la magnetización. El parámetro $\eta$ definido como $\eta=\left(d M_{r e v} / d M_{i r r}\right)_{H}$ y el parámetro móvil de Preisach $k_{m}$, se evaluaron mediante la relación $\eta=k_{m} \chi_{\text {rev }}$ para investigar el comportamiento de $M_{\text {rev }}$ Se encontró que $\eta$ no es proporcional a la susceptibilidad reversible, por lo tanto, $k_{m}$ es una función del campo interno.

Se midieron las curvas de magnetización isotérmica remanente (MIR) y de desmagnetización (DCD). A partir de los datos MIR y DCD se construyó la curva $\delta m$ y el gráfico de Henkel, el cual se encuentra por debajo de la línea de Wohlfarth indicando que domina el desorden local (efecto del tipo desmagnetizante) sobre el efecto de campo medio. Diferenciando las curvas de MIR y DCD se obtuvieron las curvas de distribución de campos de conmutación. Los valores pico de estas distribuciones de campos de conmutación difieren por un factor de 2.7.

Palabras claves: reversibilidad, remanencia, ferrita de cobalto, coprecipitación.

Reversible-irreversible magnetization processes and remanence properties of coprecipitated cobalt ferrite.

Cobalt ferrite was obtained by coprecipitation of iron (II) sulphate and cobalt (II) sulphate in acetone medium. Using the moving Preisach model, the reversible $\left(M_{r e v}\right)$ and irreversible $\left(M_{i r r}\right)$ components of the magnetization were separated. The parameter $\eta$ given by $\eta=\left(d M_{\text {rev }} / d M_{i r}\right)_{H_{i}}$ and the moving Preisach parameter $k_{m}$, were evaluated according to the relation $\eta=\mathrm{k}_{\mathrm{m}} \chi_{\mathrm{rev}}$ in order to investigate the behavior of $\mathrm{M}_{\mathrm{rev}}$. It was found that $\eta$ is not proportional to the reversible susceptibility, therefore $k_{m}$ is a function of the internal field. Isothermal remanent magnetization (IRM) and DC demagnetization (DCD) curves were measured. From these data the $\delta \mathrm{m}$ curve and the Henkel plot were obtained showing that all the points remain under the Wohlfath line indicating that the local disorder dominates (demagnetizing-like effect) over the mean-field effect. The switching field distribution curves were obtained by differentiation of the IRM and DCD curves. The peak values of these switching field distributions differ by a factor of 2.7 .

Keywords: reversibility, remanence, cobalt ferrite, coprecipitation.

Trabajo presentado en la III Reunión Iberoamericana en Materiales Electrocerámicos (México, Abril 2000).

\section{INTRODUCCIÓN}

La ferrita de cobalto es una ferrita cúbica espinela. Esta ferrita posee una estabilidad química excelente, elevada resistencia a la corrosión, anisotropía magnetocristalina, magnetoestricción y propiedades magneto-ópticas, que la hacen un material útil como medio de grabación magnética con guías de onda ópticas (1-4). Sin embargo, no se han desarrollado estudios sobre las componentes reversible e irreversible de la magnetización, ni tampoco sobre las interacciones magnéticas interpartículas. En este trabajo se estudió el proceso de magnetización para separar las componentes reversible e irreversible de la magnetización. A partir de los datos de las curvas de magnetización isotérmica remanente (MIR) y de las curvas de desmagnetización remanente DC (DCD) se obtuvo el gráfico de Henkel que se analiza en el marco del modelo de Preisach para investigar la naturaleza de las interacciones interpartículas. Se calcularon las susceptibilidades magnéticas MIR y DCD para obtener información sobre la distribución de los campos de conmutación.

\subsection{Componentes reversible e irreversible de la magnetización}

El modelo clásico de Preisach es un modelo matemático que considera el material magnético como una colección de lazos cuadrados de histéresis denominados unidades biestables de Preisach o histerones. Estas unidades de Preisach asociadas a diferentes partículas en el sistema difieren en su forma y tamaño dependiendo de parámetros tanto estructurales como magnéticos, por lo que en general se considera que la distribución de Preisach es una descripción del desorden estructural local (5)(6).

La condición necesaria y suficiente para que un material magnético pueda ser descrito por el modelo clásico de Preisach es que cumpla con las propiedades de congruencia y la propiedad de borrado descritas en la literatura (5)(6).

Los materiales ferromagnéticos obedecen la propiedad de borrado con mayor aproximación que la propiedad de congruencia. El modelo móvil de Preisach es una aproximación a la validez de la propiedad de congruencia, considerando que el campo asociado con cada partícula en estudio, es un campo 
efectivo $H_{e f^{\prime}}$ dado por (5)(6)(7):

$$
H_{e f}=H_{i}+k_{m} M=H_{i}+k_{m}\left(M_{r e v}+M_{i r r}\right)
$$

donde $H_{i}$ es el campo interno definido por $H_{i}=H_{a p}-N_{d} \mathrm{M}$, siendo $H_{a p}$ el campo aplicado, $N_{d}$ el factor de desmagnetización y $M$ la magnetización total. $k_{m}$ es el llamado parámetro móvil de Preisach. El campo medio $k_{m} M$ es un campo proveniente de considerar el efecto que tienen sobre una partícula determinada, las partículas de su entorno y promedia estos efectos a gran escala.

Cammarano, Mc. Cormick y Street [8], así como Cornejo y Misell [9] desarrollaron recientemente investigaciones sobre la componente reversible de la magnetización, para estudiar los procesos elementales en los mecanismos de inversión de la magnetización. Cammarano, McCormick y Street (8), propusieron que la magnetización reversible $M_{\text {rev }}$, no solo es función del campo interno, sino también de la magnetización irreversible lo cual se expresa por la ecuación constitutiva:

$$
d M_{\text {rev }}=\chi_{\text {rev }} d H_{i}+\eta d M_{i r r}
$$

donde $M_{\text {tot }}$ es la magnetización total y $\chi_{\text {rev }}$ es la susceptibilidad magnética reversible definida por:

$$
\chi_{\text {rev }}=\left(d M_{r e v} / d H_{i}\right)_{M_{i r r}}
$$

y $\eta$ es un parámetro definido por

$$
\eta=\left(d M_{\text {rev }} / d M_{i r r}\right)_{H_{i}}
$$

Usando el modelo móvil de Preisach (9)(10), Cornejo y Missell demostraron que existe una relación directa entre $\eta$ y $\chi_{\text {rev }}$ dada por:

$$
\eta=k_{m} \chi_{\text {rev }}
$$

Considerando que $d M_{i r r}=\chi_{i r r} d H_{i^{\prime}}$ obtenemos:

$$
d M_{\text {rev }}\left(H_{i}\right)=\chi_{\text {rev }}\left(1+k_{m} \chi_{i r}\right) d H_{i}
$$

La magnetización reversible puede determinarse integrando la ecuación (6) entre $0 \mathrm{y} \mathrm{H}_{\mathrm{i}}$, a lo largo de la curva inicial de magnetización y de la curva de desmagnetización:

$$
M_{\text {rev }}\left(H_{i}\right)=\int_{0}^{H_{i}} \chi_{\text {rev }}\left(H_{i}\right)\left[1+k_{m} \chi_{i r r}\left(H_{i}\right)\right] d H_{i}
$$

La magnetización irreversible vendrá dada por:

$$
M_{i r r}\left(H_{i}\right)=M_{t o t}\left(H_{i}\right)-M_{r e v}\left(H_{i}\right)
$$

En este trabajo se determinó la magnetización reversible e irreversible, encontrándose una correlación entre el método DCD (11) para determinar $M_{r e v}$ y $M_{i r r}$, y el modelo móvil de Preisach usado por Cornejo y Missell (9)(10).

\subsection{Propiedades de remanencia}

El modelo de Preisach de histéresis magnética es una teoría general que permite estudiar las interacciones magnéticas (12), ya que las suposiciones que limitan la validez de la teoría de Wohlfarth no están presentes en el marco del modelo de Preisach (5). Para describir las interacciones magnéticas se utilizan gráficos de Henkel y también los gráficos de $\delta m$ en función del campo magnético aplicado. Sobre esta base pueden estudiarse las interacciones entre partículas no uniaxiales, como es el caso de la ferrita de cobalto cúbica espinela estudiada.

Los tres estados de remanencia magnetizante $\left(M_{r}\right)$, remanencia desmagnetizante $\left(M_{d}\right)$ y remanencia de saturación $\left(M_{\infty}\right)$ están asociados con tres particiones del plano de Preisach (5). El gráfico de Henkel muestra la dependencia de $M_{d}(H) \operatorname{con} M_{r}(H)$. Considerando una distribución de Preisach concentrada a lo largo del eje $h_{c}\left(h_{u}=0\right)$, se obtiene un límite superior para el gráfico de Henkel dado por la relación $M_{d}=M_{\infty}-2 M_{r}$ (5). Por otro lado, si consideramos una distribución de Preisach concentrada alrededor de una coercitividad local $h_{c}=h_{0}$, se obtiene un límite inferior para el gráfico de Henkel dado por $M_{d}=-M_{r}$ (12). Ambos límites conducen a las desigualdades siguientes: $-M_{r} \leq M_{d} \leq M_{\infty}-2 M_{r}$ (12). En el estudio de los efectos de interacción se busca con frecuencia no linealidades del comportamiento de $M_{d}$ vs $M_{r}$. Las interacciones con $M_{d}>(<) M_{\infty}-2 M_{r}$, tienen una naturaleza magnetizante (demagnetizante) (5)(13).

\section{PROCEDIMIENTO EXPERIMENTAL}

Se preparó una solución $1 \mathrm{M}$ de sulfato de cobalto y sulfato de hierro en medio acuoso, en la cual la proporción Fe/Co fue de $2 / 1$. Esta solución fue rápidamente añadida a $60 \mathrm{ml}$ de acetona bajo agitación contante, y se obtuvo un precipitado color rosa, que fue filtrado y lavado con agua varias veces.

Este precipitado se secó durante $4 \mathrm{~h}$ a una temperatura de $80^{\circ} \mathrm{C}$ y posteriormente se calcinó a $1100^{\circ} \mathrm{C}$ durante $3 \mathrm{~h}$, formándose la ferrita de cobalto $\mathrm{CoFe}_{2} \mathrm{O}_{4}$.

$\mathrm{El}$ análisis estructural del producto coprecipitado fue realizado por espectroscopia infrarroja (IR), para lo cual se usó un espectrómetro infrarrojo Nicolet Magna, en un rango de número de onda desde 4000 hasta $400 \mathrm{~cm}^{-1}$.

El producto seco fué analizado estructuralmente mediante difracción de rayos $X$ (DRX), utilizando un difractómetro de rayos $X$ Siemens D-5000, con radiación CuKa, en un intervalo angular $2 \mathrm{q}$ desde $5^{\circ}$ hasta $80^{\circ}$, con un paso de $1^{\circ}$. El estudio estructural de la ferrita de cobalto obtenida después de la calcinación también fue realizado por difracción de rayos $X$, pero en este caso el intervalo angular $2 q$ fue desde $5^{\circ}$ hasta $120^{\circ}$, con un paso de $0.05^{\circ}$, y el espectro obtenido fue refinado usando el método de Rietveld.

La microestructura de las partículas de ferrita de cobalto fue estudiada por microscopía electrónica de transmisión, para lo cual se utilizó un microscopio electrónico de transmisión Phillips CM-200 acoplado con un analizador de rayos $\mathrm{X}$ dispersados EDAX DX-4.

El estudio magnético fue llevado a cabo a temperatura ambiente, utilizando un magnetómetro vibracional LDJ Electronics 9600, el cual permite ser programado a través del software que presenta, utilizando el lenguaje TDL (Test of Development Language).

Para determinar las componentes reversibles e irreversibles de la magnetización la muestra en estado desmagnetizado se lleva al estado de saturación positivo y después se invierte el sentido del campo magnético hasta un determinado campo interno $H_{i}$ negativo, donde permanece por un espacio de tiempo de 100 segundos, para permitir un acercamiento al equilibrio termodinámico retardado por el fenómeno de viscosidad magnética. Posteriormente se incrementa hasta cero el valor de campo interno, con un paso de $10 \mathrm{Oe}$, generándose la curva ascendente del ciclo de retroceso. Desde campo interno cero, se retorna al mismo campo negativo $H_{i}$ 
completándose el ciclo de retroceso. La muestra es llevada a saturación y se repite nuevamente el proceso para otro valor del campo interno $H_{i}$. Los valores utilizados de campo interno $H_{i}$ decrecen con un paso de $10 \mathrm{Oe}$, hasta alcanzar un campo interno de -1200 Oe.

La magnetización irreversible $M_{r r i}$ de un ciclo de retroceso es igual al valor de la magnetización total cuando se completa la curva ascendente del ciclo retroceso a campo interno cero. Este valor de la magnetización irreversible $M_{r r i}$ permanece prácticamente constante para todo el ciclo de retroceso dado y, para un determinado valor de campo a lo largo de la curva ascendente del ciclo de retroceso, la magnetización total $M_{\text {tot }}$ es la suma de esta magnetización irreversible más la reversible. De otra forma, la magnetización reversible $M_{\text {rev }}$ es la diferencia de estas dos magnetizaciones $\left(M_{r e v}=M_{t o t}-M_{r r i}\right)$.

Para medir las propiedades de remanencia de la ferrita de cobalto se preparó una muestra en forma de disco sin prensar y se sinterizó a $1100^{\circ} \mathrm{C}$ por 3 horas. Como aglutinante se utilizó una cantidad equivalente al $4 \%$ del peso de la ferrita de cobalto de alcohol polivinílico al $10 \%$.

Curva de Magnetización Isotérmica Remanente (MIR): A partir del estado desmagnetizado en AC, se aplica un campo positivo $h$ que posteriormente se quita. La magnetización remanente que queda con campo aplicado cero es la magnetización isotérmica remanente $M_{r}(h)$. Esta magnetización es la componente irreversible del proceso. Este procedimiento se realizó variando el campo aplicado con un paso de 50 Oe hasta que se alcanzó el estado de saturación en 10 kOe.

$M_{\infty}$ es el valor de la magnetización isotérmica remanente cuando se aplica un campo que satura la muestra.

Curva de desmagnetización remanente en corriente directa (curva remanente DCD): A partir del estado de saturación, se aplica un campo negativo - $h$ que posteriormente se quita. La magnetización remanente que queda con campo aplicado cero es la magnetización DCD remanente $M_{d}(h)$. Esta magnetización es la componente irreversible del proceso. Este procedimiento se realizó variando el campo aplicado con un paso de -50 Oe hasta que se alcanzó el estado de saturación negativo en $-10 \mathrm{kOe}$.

En la literatura (5)(6), los valores de $M_{r}(h)$ y $M_{d}(h)$ son reportados en función del módulo del campo aplicado por lo que en los gráficos de remanencia solo aparecen valores positivos de campo.

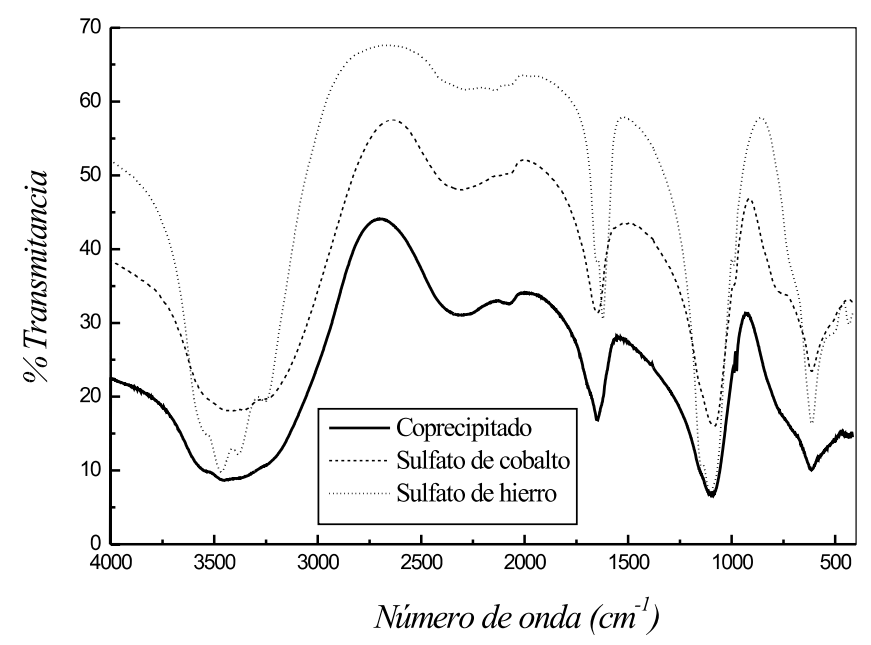

La curva de Henkel se construye graficando $M_{d}(h) / M_{\infty}$ en función de $M_{r}(h) / M_{\infty}$. La curva $\delta m$ se construye graficando los valores generados por la diferencia $M_{d}(h) / M_{\infty}-\left[M_{\infty}-2 M_{r}(h)\right]$ en función del campo aplicado $h$. Finalmente se obtuvieron las curvas de susceptibilidades MIR y DCD derivando las curvas $M_{r}(h)$ y $M_{d}(h)$ con respecto al campo aplicado $h$, respectivamente (5)(6)(13).

\section{RESULTADOS Y DISCUSIÓN}

En la Figura 1 se muestran los espectros infrarrojo de absorción de los reactivos precursores y del producto coprecipitado lavado y antes de ser tratado térmicamente. En los tres espectros aparecen dos bandas una entre $610-680 \mathrm{~cm}^{-1}$ y la otra entre $1080-1130 \mathrm{~cm}^{-1}$ correspondientes a las vibraciones de enlace presentes en los aniones sulfatos $\mathrm{SO}_{4}{ }^{\prime}$ (14) En estos espectros también pueden apreciarse dos bandas, una entre $1600-1800$ $\mathrm{cm}^{-1}$ y la otra entre 3000-3600 $\mathrm{cm}^{-1}$ correspondientes a los modos de "alargamiento" y "flexión respectivamente de la molécula de agua (14). De este espectro puede observarse que no aparece ninguna banda que determine modos vibracionales de un nuevo enlace entre las moléculas de sulfato de hierro y sulfato de cobalto. Por otro lado en la Figura 2, se muestra el espectro DRX, del producto coprecipitado seco, el cual corresponde a una mezcla de sulfatos con diferentes grados de hidratación, esto es $\mathrm{CoSO}_{4} \cdot 4 \mathrm{H}_{2} \mathrm{O}(15) \mathrm{FeSO}_{4}(\mathrm{OH})$ [16] y $\mathrm{FeSO}_{4} \cdot \mathrm{H}_{2} \mathrm{O}$ (17). Como puede verse a partir del análisis de IR y DRX, las sales iniciales no reaccionan con el medio acetona, ocurriendo una precipitación conjunta, cuyo precipitado contiene una mezcla íntima de las sales de sulfato de cobalto y sulfato de hierro.

El espectro DRX del producto calcinado se muestra en la Figura 3. Este espectro fue refinado usando el método de Rietveld, obteniéndose una estrucutra cúbica espinela correspondiente a la ferrita de cobalto con un parámetro de celda $\mathrm{a}=8.3763(4) \AA ̊$ y un parámetro de oxígeno $\mathrm{m}=0.3821$ (8) $\mathrm{A}$. Estos datos son comparables a los reportados en la bibliografía (18).

Se realizó un promedio del tamaño de las partículas de polvo de ferrita de cobalto, medidas a partir de varias imágenes de MET, obteniéndose un tamaño alrededor de $350 \mathrm{~nm}$. En la Figura 4 se muestra una de las partículas de ferrita en la que se observa la forma poliedrica de ellas debido a un crecimiento en facetas.

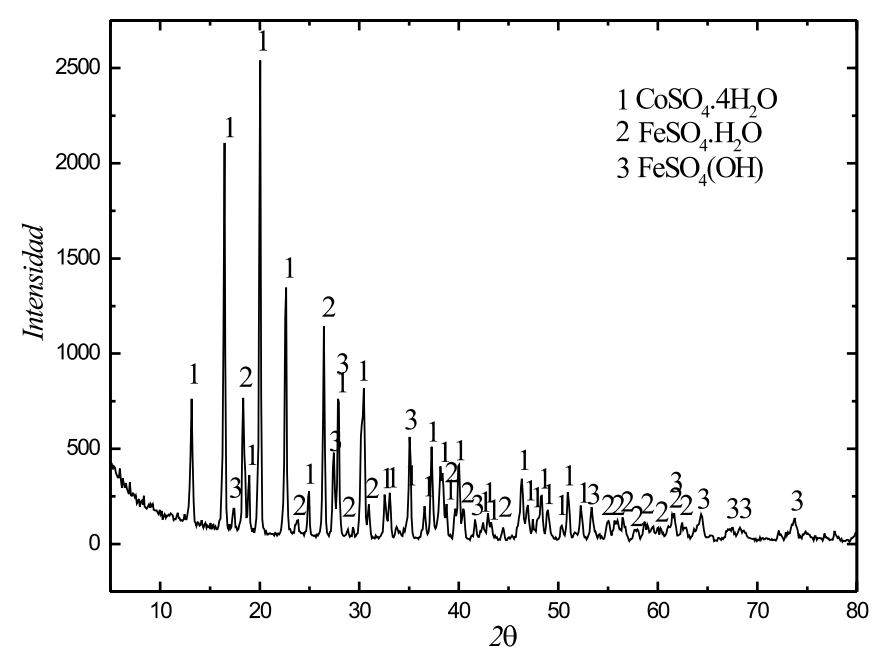




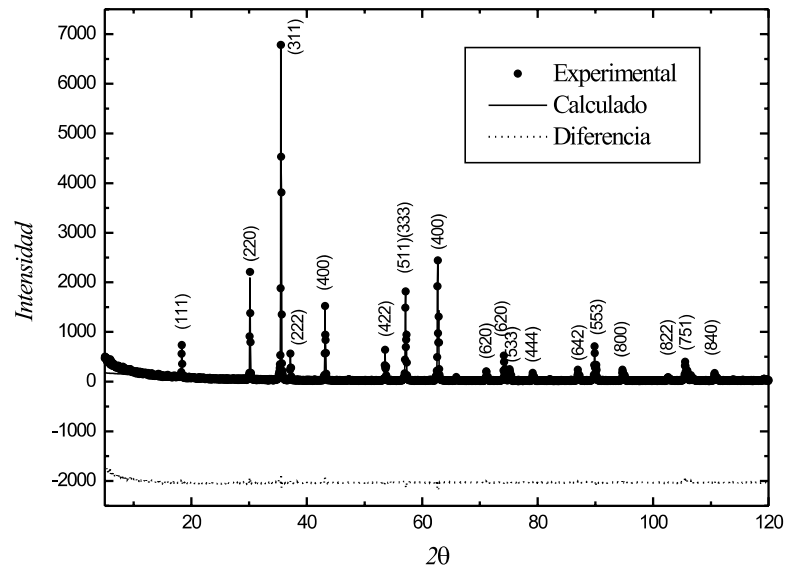

Figura 3 Patrón de difracción de rayos $\mathrm{X}$ refinado por el método de Rietveld, del producto en polvo de ferrita de cobalto obtenido luego de la calcinación.

\section{- Componentes de la magnetización:}

Se tomaron 120 curvas de retroceso para producir valores de $M_{\text {rev }}$ vs $M_{i r r}$ a valores de campo interno constante. Estas curvas tomadas desde 0 Oe hasta -1200 Oe fueron ajustadas utilizando un polinomio de segundo grado que posteriormente fue diferenciado y evaluado en el valor de campo que corresponde al punto inicial de la curva de retroceso, común al lazo mayor de histéresis. Usando la ecuación (4) se obtuvo el parámetro $\eta$. Por otro lado $\chi_{\text {rev }}$, fue obtenido utilizando la ecuación (3) a partir de los datos obtenidos para $M_{\text {rev }}$ según el método DCD.

La Figura 5a muestra el comportamiento de $\eta$ y $\chi_{\text {rev }}$ con el campo interno $H_{i} \cdot \eta$ es inicialmente positiva, presentando dos máximos, uno alrededor de -500 Oe y el otro alrededor de 1100 Oe. El hecho de que exista un máximo desplazado del cero de campo interno en la curva $\chi_{\text {rev }}$ vs $H_{i^{\prime}}$ indica que la componente reversible de la magnetización es histérica, lo que concuerda con lo reportado por Cornejo y Missell (10).

La parte del ciclo mayor de histéresis entre 0 Oe y -1200 Oe de campo interno fue ajustada mediante un polinomio de grado 9, diferenciada y evaluada en valores de campo que corresponden al inicio de cada curva de retroceso, con la finalidad de obtener los valores de susceptibilidad total, $\chi_{\text {tot }}$, en dichos puntos. El mismo procedimiento de ajuste fue realizado a lo largo de cada curva de retroceso para determinar $\chi_{\text {rev }}$. La susceptibilidad irreversible $\chi_{i r r}$ se obtiene mediante la diferencia $\left(\chi_{\text {tot }}-\chi_{\text {rev }}\right)$.

El parámetro $k_{m}$ se obtuvo dividiendo los valores de $\eta$ entre los valores de $\chi_{\text {rev }}$, de acuerdo a la ecuación (5). La Figura $5 \mathrm{~b}$ muestra el comportamiento de $k_{m}$ vs $H_{i}$. Como puede observarse esta gráfica nos indica que $k_{m}$ es una función del campo interno, lo que concuerda con los resultados obtenidos por Crew, Woodward y Street (11).

La función $\chi_{\text {rev }}\left(H_{i}\right)\left[1+k_{m} \chi_{i r r}\left(H_{i}\right)\right]$ fue evaluada y numéricamente integrada con la finalidad de obtener $M_{\text {rev }} ; M_{i r r}\left(H_{i}\right)$ fue posteriormente calculado mediante la ecuación (8). La Figura 6 muestra las componentes reversibles e irreversibles de la magnetización total considerando que el parámetro $\mathrm{k}_{\mathrm{m}}$ es una función del campo interno. Si el presente estudio se realizara sobre la curva inicial de magnetización, la componente $M_{\text {rev }}\left(H_{i}\right)$ se desplazaría de cero sobre el eje de magnetización, como lo demuestran Cornejo y Missell (9)(10).

- Propiedades de remanencia:

En las Figuras $7 \mathrm{a}$ y $7 \mathrm{~b}$ se muestran las magnetizaciones y susceptibilidades remanentes DCD y MIR, respectivamente.

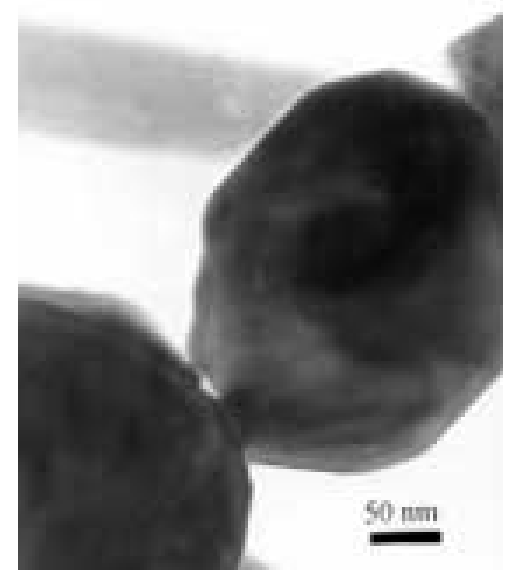

Figura 4 Micrografía electrónica de transmisión de una partícula de ferrita de cobalto obtenida por coprecipitación.
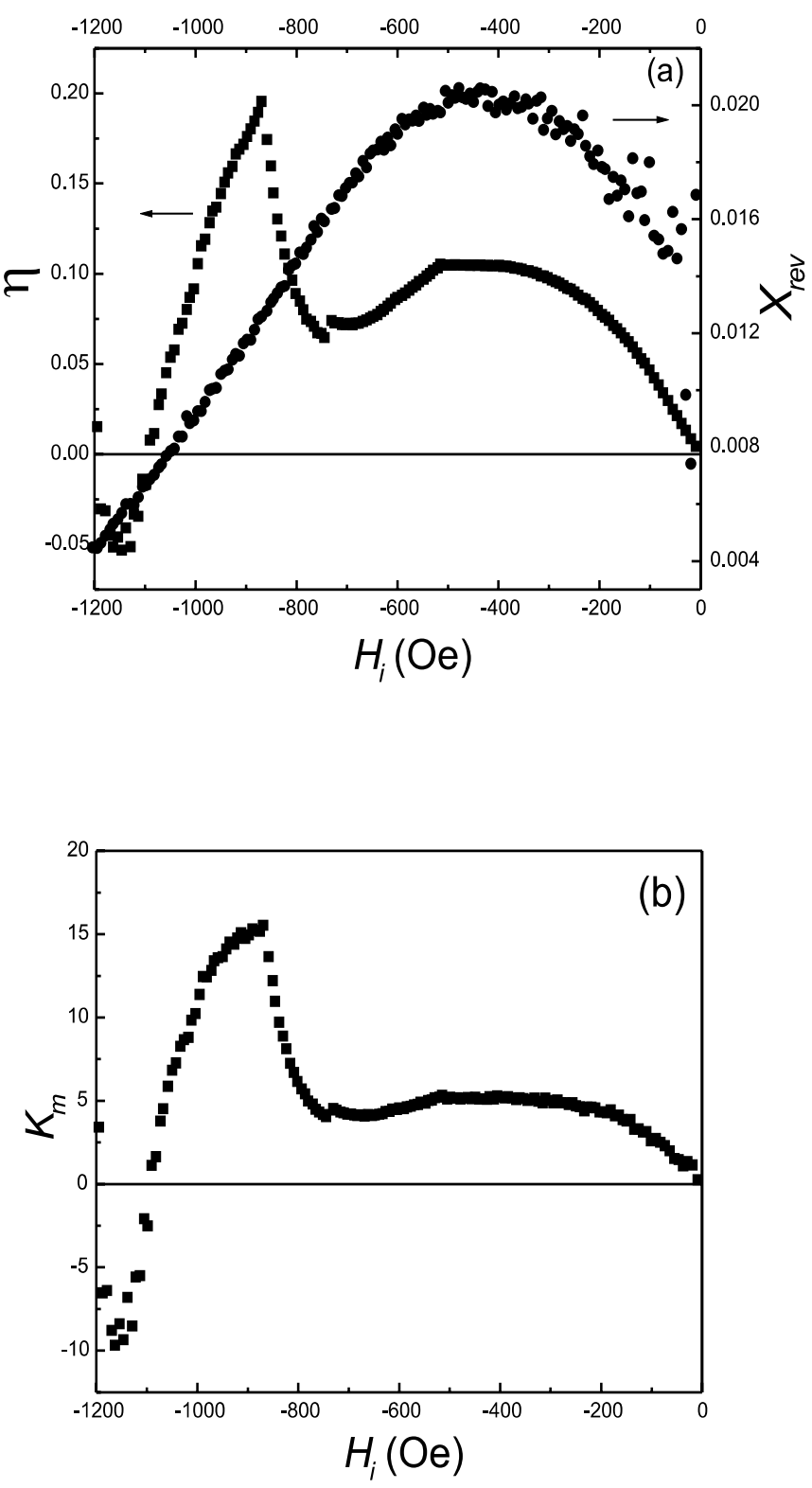


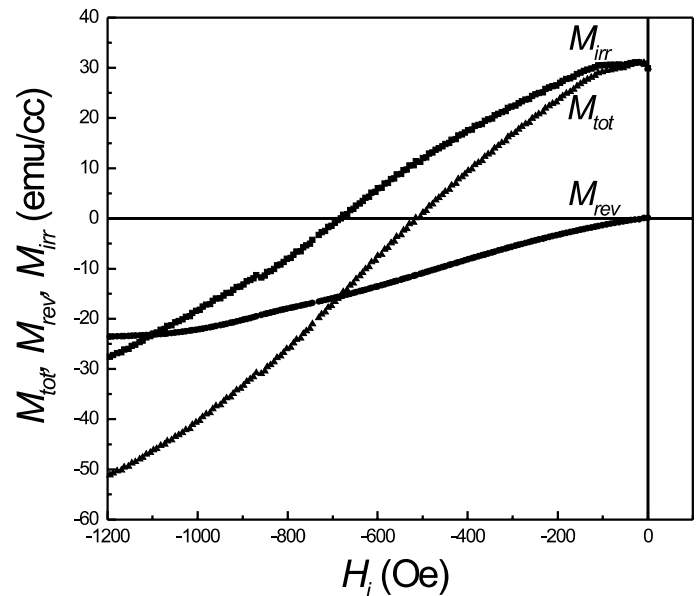

Figura 6 Curvas de magnetización total y sus componenetes reversibles e irreversibles, obtenidas por el método de Cornejo y Missell [7][9].
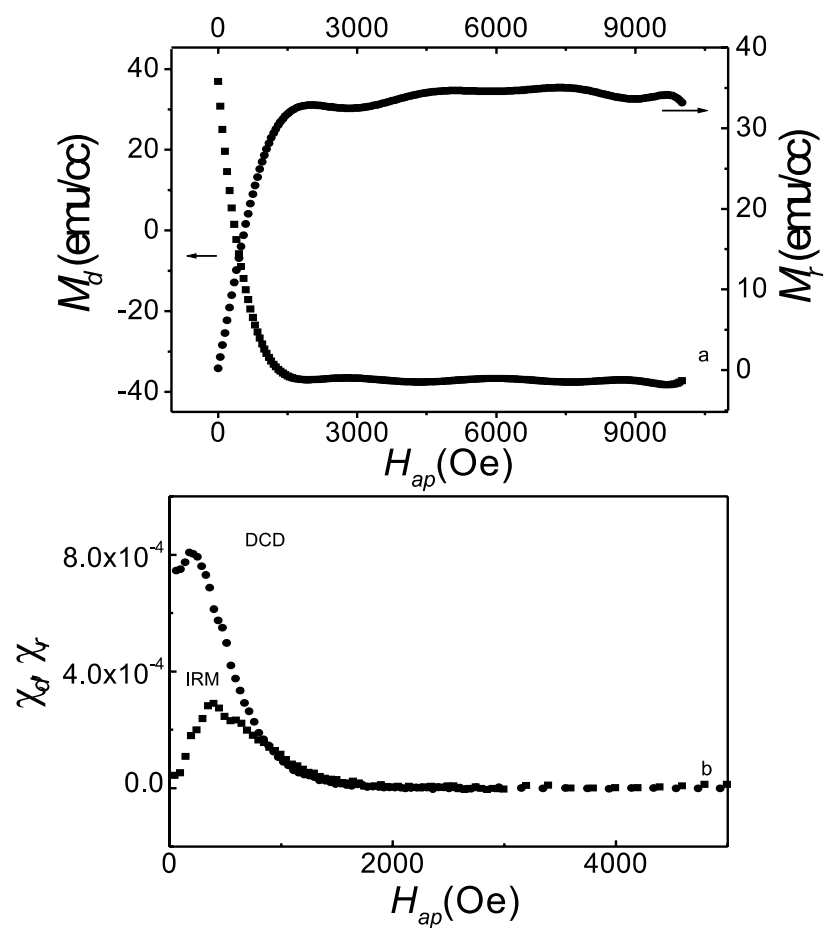

Figura 7 (a) Curvas de magnetización y (b) susceptibilidades remanentes DCD y MIR en función del campo aplicado.



De acuerdo a la relación de Wolrfarth para partículas que no interaccionan, la relación de amplitudes de los picos en las curvas de susceptibilidades $\chi_{D C D}$ y $\chi_{M I R}$, obtenidas a partir de la diferenciación de los valores de magnetización respectivos, debe cumplir una proporción 1:2. Sin embargo en nuestro caso, esta relación es 2.7, lo cual indica la presencia de interacciones, cuya naturaleza se estudia a continuación.

Se procesaron los datos de manera que se obtuvieron curvas $\delta m$ y gráficos de Henkel. En el caso de que no se presenten interacciones entre las partículas, el gráfico $\delta m$ debe ser una línea horizontal en $\delta m=0$. La Figura 8, muestra valores negativos de $\delta m$, lo cual indica efectos de interacción de naturaleza desmagnetizante (5)(6)(13). En efecto, el campo magnético promedio que siente una partícula es en el sentido contrario a la dirección del campo aplicado.

La Figura 9 muestra el gráfico de Henkel, observándose que para todo el rango de campos, la curva pasa por debajo de la línea de Wohlfarth. Aun en el marco del modelo móvil de Preisach, el hecho de que el ploteo de Henkel no se ajuste a la línea de Wohlfarth indica la presencia de interacciones. Sin embargo a partir del hecho de que la curva experimental pasa por debajo de la línea de Wohlfarth y considerando que $k_{m}>0$ para campos mayores a -1100 Oe, como se observa en la Figura 6 , se concluye que en el sistema domina el desorden estructural local sobre los efectos de campo medio entre las partículas (5).

\section{CONCLUSIONES}

Se usó el método DCD y el modelo móvil de Preisach para describir las componentes reversible e irreversible de la magnetización de la $\mathrm{CoFe}_{2} \mathrm{O}_{4}$ obtenida por coprecipitación. El máximo de la curva $\chi_{\text {rev }}$ vs $H_{i}$ desplazado del origen, indica un comportamiento histérico de la magnetización reversible. La función de interrelación $\eta$ entre $M_{r e v}$ y $M_{i r r}$ no es proporcional a $\chi_{\text {rev }}$. El parámetro móvil $\mathrm{k}_{\mathrm{m}}$ que relaciona $\eta$ y $\chi_{\text {rev }}$ es una función del campo interno, lo que tiene consecuencias sobre el campo medio, que describe las interacciones en dicho modelo.

La interacción entre las partículas es del tipo desmagnetizante, según la curva $\delta m$.

El gráfico de Henkel de los datos MIR y DCD se analizó en el marco del modelo móvil de Preisach. Los datos experimentales se encuentran por debajo de la línea de Wohlfarth e indican un desorden local dominante sobre los efectos de campo medio para campos en los cuales $k_{m}>0$. Los máximos de las curvas de distribución de campo de conmutación MIR y DCD no coinciden y sus valores difieren por un factor de 2.7 .

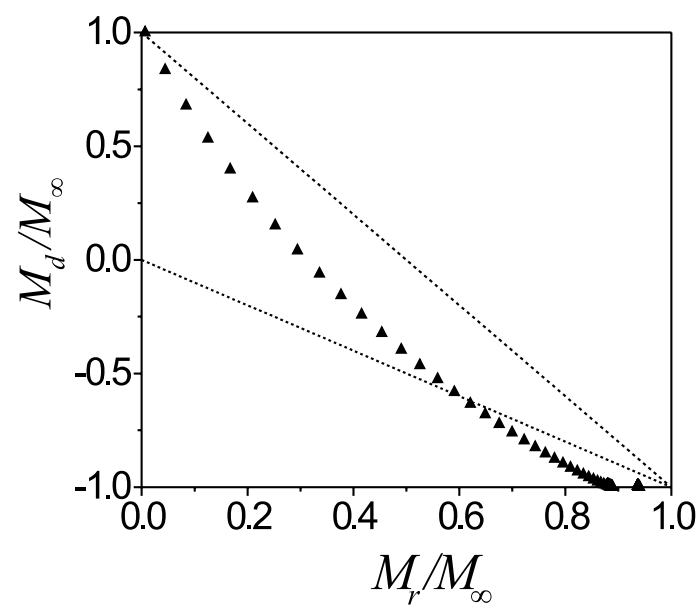




\section{BIBLIOGRAFÍA}

1. M. Grigorova, H. J. Blythe, V. Blaskov, V. Rusanov, V. Petkov, V. Masheva, D. Nihtianova, LI. M. Martinez, J. S. Muñoz, M. Mikhov, Magnetic properties and Mössbauer spectra of nanosized $\mathrm{CoFe}_{2} \mathrm{O}_{4}$ powders, J. Magn. Magn. Mater., $183,(1998), 163-172$

2. Shino N. Okuno, Susumu Hashimoto, and Koichiro Inomata, Preferred crystal orientation of cobalt ferrite thin films induced by ion bombardment during deposition, J. Appl. Phys. 71, 12 (1992).

3. D. H. Lee, H. S. Kim, J. Y. Lee, Ch. H. Yo and K. H. Kim, Characterization of the magnetic properties and transport mechanism of $\mathrm{Co}_{x} \mathrm{Fe}_{3-x} \mathrm{O}_{4}$ spinel, Solid State communications, 96, No. 7, (1995), 445-449.

4. Fuxiang Cheng, Zuogan Peng, Chunsheng Liao, Zhigang $\mathrm{Xu}$, Song Gao, Chunhua Yan, Dongjan Wang and Jing Wang, Chemical synthesis and magnetic study of nanocrystalline thin films of cobalt spinel ferrites, Solid State Communications, Vol. 107, No. 9, pp. 471-476 (1998).

5. Giorgio Bertotti, Hysteresis in Magnetism, Academic Press, p. 488-493, (1998). 6. Edward Della Torre, Magnetic Hysteresis, IEEE Press, New York(1999).

7. M. Emura, A. C. Neiva and F. P. Missell, Hybrid Magnets, Mat. Sci. Forum, 284-288 (1999)

8. R. Cammarano, P. G. McCormick and R. Street, The interrelation of reversible and irreversible magnetization, J. Phys. D 29, 2327 (1996).
9. D.R. Cornejo and F.P. Missell, Study of Reversible and Irreversible Magnetization Processes using the Moving Preisach Model, Mat. Sci. Forum, 302-303, 53-58 (1999).

10. D.R. Cornejo and F.P. Missell, Application of Preisach model to nanocrystalline magnets, J. Magn. Magn. Mater., 203, 41-45 (1999).

11. D. C. Crew, P. G. McCormick and R. Street, An investigation of reversible magnetization in NdFeB, J. Appl. Phys. 86, No. 6, 3278-3284 (1999).

12. V. Basso and G. Bertotti, Description of magnetic interactions and Henkel plots by Preisach hysteresis model, IEEE Transactions on magnetics, Vol. 30, No1, p. 64-72, (1994).

13. M. El Hilo, K. O'Grady and R. W. Chantrell, Interaction effects and activation volumes in Barium ferrite recording media, IEEE Transactions on magnetics, Vol. 27, No. 6, p. 4666-4668, (1991).

14. Foil A. Miller and Charles H. Wilkins. Analytical Chemistry, Vol. 4, p. 1253 (1952)

15. Cartoteca PDF JCPDS-ICDD 14-488 (1996).

16. Cartoteca PDF JCPDS-ICDD 16-939 (1996).

17. Cartoteca PDF JCPDS-ICDD 45-1365 (1996).

18. N. W. Grimes, Off-centre Ions in Compounds with Spinel Structure, Philos. Mag, 1217 (1972)

Recibido: 31.07 .00

Aceptado: 15.01 .01

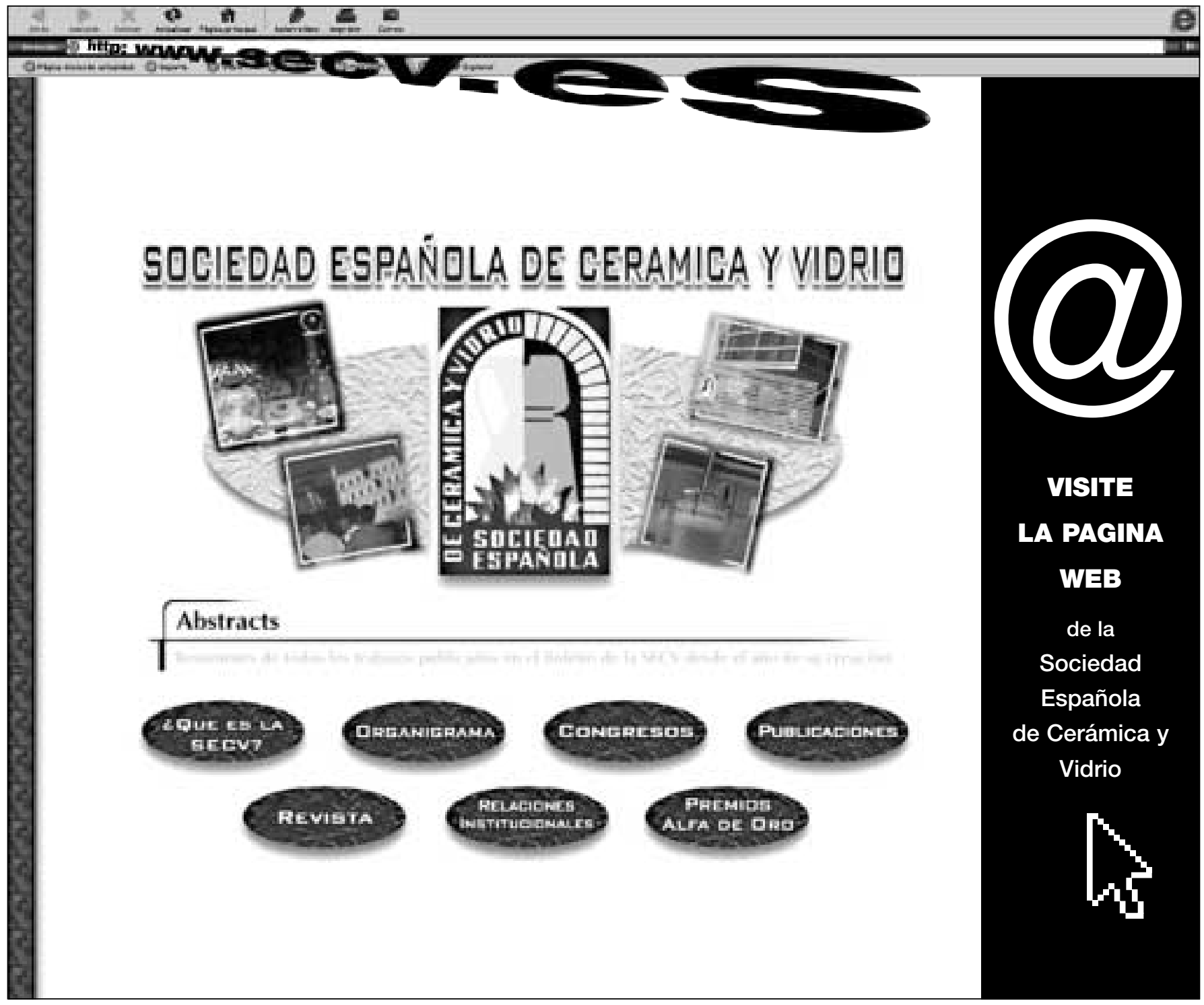

